\title{
Dynamic Changes of Antibodies to SARS-CoV-2 in COVID-19 Patients at Early Stage of Outbreak
}

\author{
Huaqing Shu ${ }^{1,2} \cdot$ Shuzhen Wang ${ }^{3} \cdot$ Shunan Ruan ${ }^{3} \cdot$ Yaxin Wang ${ }^{1,2} \cdot$ Jiancheng Zhang ${ }^{1,2} \cdot$ Yin Yuan ${ }^{1,2}$. \\ Hong Liu ${ }^{1,2,3} \cdot$ Yongran $\mathrm{Wu}^{1,2,3} \cdot$ Ruiting $\mathrm{Li}^{1,2} \cdot$ Shangwen $\mathrm{Pan}^{1,2} \cdot$ Yaqi Ouyang ${ }^{1,2} \cdot$ Shiying Yuan ${ }^{1,2,3}$. \\ Peng Zhou ${ }^{4}$ (D) You Shang ${ }^{1,2,3}$
}

Received: 11 May 2020/Accepted: 15 June 2020 / Published online: 27 July 2020

(C) Wuhan Institute of Virology, CAS 2020, corrected publication 2020

\begin{abstract}
The coronavirus disease 2019 (COVID-19), caused by SARS-CoV-2, has spread around the world with high mortality. To diagnose promptly and accurately is the vital step to effectively control its pandemic. Dynamic characteristics of SARSCoV-2-specific antibodies which are important for diagnosis of infection have not been fully demonstrated. In this retrospective, single-center, observational study, we enrolled the initial 131 confirmed cases of COVID-19 at Jin-Yin-Tan Hospital who had at least one-time antibody tested during their hospitalization. The dynamic changes of $\operatorname{IgM}$ and IgG antibodies to SARS-CoV-2 nucleocapsid protein in 226 serum samples were detected by ELISA. The sensitivities of IgM and IgG ELISA detection were analyzed. Result showed that the sensitivity of the IgG ELISA detection (92.5\%) was significantly higher than that of the $\operatorname{IgM}(70.8 \%)(P<0.001)$. The meantimes of seroconversion for IgM and $\operatorname{IgG}$ were 6 days and 3 days, respectively. The IgM and IgG antibody levels peaked at around 18 days and 23 days, and then IgM fell to below the baseline level at about day 36, whereas IgG maintained at a relatively high level. In conclusion, antibodies should be detected to aid in diagnosis of COVID-19 infection. IgG could be a sensitive indicator for retrospective diagnosis and contact tracing, while IgM could be an indicator of early infection.
\end{abstract}

Keywords Severe acute respiratory syndrome coronavirus 2 (SARS-CoV-2) · Coronavirus disease 2019 (COVID-19) . Coronavirus $\cdot$ Antibody $\cdot$ Serology

Huaqing Shu and Shuzhen Wang contributed equally to this work.

You Shang

you_shanghust@163.com

$\triangle$ Peng Zhou

peng.zhou@wh.iov.cn

1 Department of Critical Care Medicine, Union Hospital, Tongji Medical College, Huazhong University of Science and Technology, Wuhan 430022, China

2 Institute of Anesthesiology and Critical Care Medicine, Union Hospital, Tongji Medical College, Huazhong University of Science and Technology, Wuhan 430022, China

3 Jin-Yin-Tan Hospital, Wuhan 430023, China

4 CAS Key Laboratory of Special Pathogens, Wuhan Institute of Virology, Center for Biosafety Mega-Science, Chinese Academy of Sciences, Wuhan 430071, China

\section{Introduction}

Since December 2019, severe acute respiratory syndrome coronavirus 2 (SARS-CoV-2) pneumonia has spread rapidly in China and soon after around the world. It has been declared by WHO as a global public health emergency and named as Coronavirus disease 2019 (COVID-19) on January 13, 2020 (Huang et al. 2020; Zhou et al. 2020b; Zhu et al. 2020). As of July 08, 2020, there have been about 11,669,259 confirmed cases, and 539,906 deaths caused by the COVID-19 (WHO 2020a).

The clinical spectrum of patients with COVID-19 varies from asymptomatic infection, mild discomfort to severe viral pneumonia with respiratory failure and even death (Chen et al. 2020; Wang et al. 2020). The symptoms and clinical features of patients with COVID-19 include lower respiratory tract illness with fever, dry cough and dyspnea. These symptoms are similar to those of two other diseases caused by coronaviruses: severe acute respiratory 
Table 1 Demographics and clinical characteristics of the included patients.

\begin{tabular}{|c|c|}
\hline Clinical characteristics & $(\mathrm{n}=131)$ \\
\hline Sex (Male/Female) & $90(68.7 \%) / 41(31.3 \%)$ \\
\hline Age, years & $51.4 \pm 11.8(24-81)$ \\
\hline \multicolumn{2}{|l|}{ Age range, years } \\
\hline 24-39 & $19(14.5 \%)$ \\
\hline $40-59$ & $76(58.0 \%)$ \\
\hline $60-79$ & $35(16.7 \%)$ \\
\hline$\geq 80$ & $1(0.8 \%)$ \\
\hline Days from onset to hospitalization & $9.04 \pm 3.93(1-24)$ \\
\hline Time of staying in hospital (days) & $18.26 \pm 10.06(4-72)$ \\
\hline Normal/severe/critical cases & $15(11.4 \%) / 82(62.6 \%) / 34(26.0 \%)$ \\
\hline Comorbidities & $55(42 \%)$ \\
\hline Hypertension & $31(23.7 \%)$ \\
\hline Diabetes mellitus & $14(10.7 \%)$ \\
\hline Heart disease & $7(5.3 \%)$ \\
\hline \multicolumn{2}{|l|}{ Epidemiologic history } \\
\hline History of residence or travel & $131(100 \%)$ \\
\hline Exposure to Huanan Seafood Wholesale Market & $75(57.3 \%)$ \\
\hline History of contacting with COVID-19 patients & $12(9.2 \%)$ \\
\hline Clustered onset & $9(6.9 \%)$ \\
\hline \multicolumn{2}{|l|}{ Onset symptoms } \\
\hline Fever & $124(94.7 \%)$ \\
\hline Cough & $96(73.3 \%)$ \\
\hline Dyspnea & $50(38.2 \%)$ \\
\hline Fatigue & $43(32.8 \%)$ \\
\hline Shortness of breath & $33(25.2 \%)$ \\
\hline Gasping & $22(16.8 \%)$ \\
\hline Muscle ache & $20(15.3 \%)$ \\
\hline Headache & $15(11.5 \%)$ \\
\hline Chill & $12(9.2 \%)$ \\
\hline Chest pain & $7(5.3 \%)$ \\
\hline Nausea & $5(3.8 \%)$ \\
\hline Dizziness & $5(3.8 \%)$ \\
\hline Sore throat & $4(3.1 \%)$ \\
\hline Runny nose & $4(3.1 \%)$ \\
\hline Difficulty breathing & $4(3.1 \%)$ \\
\hline Joint soreness & $4(3.1 \%)$ \\
\hline Palpitations & $3(2.3 \%)$ \\
\hline Vomit & $3(2.3 \%)$ \\
\hline Shivering & $3(2.3 \%)$ \\
\hline Diarrhea & $2(1.5 \%)$ \\
\hline \multicolumn{2}{|l|}{ Treatment } \\
\hline Glucocorticoids & $64(48.9 \%)$ \\
\hline Immunoglobulin & $19(14.5 \%)$ \\
\hline High-flow Nasal Cannula & $33(25.2 \%)$ \\
\hline Non-invasive ventilation & $16(12.2 \%)$ \\
\hline Invasive ventilation & $7(5.3 \%)$ \\
\hline Extracorporeal membrane oxygenation & $4(3.1 \%)$ \\
\hline Renal replacement therapy & $6(4.6 \%)$ \\
\hline Blood transfusion & $4(3.1 \%)$ \\
\hline Vasoconstrictive agents & $4(3.1 \%)$ \\
\hline
\end{tabular}


Table 1 continued

\begin{tabular}{ll}
\hline Clinical characteristics & $(\mathrm{n}=131)$ \\
\hline Complication & $72(55.0 \%)$ \\
Liver dysfunction & $48(36.6 \%)$ \\
Acute respiratory distress syndrome & $40(30.5 \%)$ \\
Hypoproteinemia & $34(26.0 \%)$ \\
Sepsis & $18(13.7 \%)$ \\
Thrombocytopenia & $16(12.2 \%)$ \\
Acute kidney injury & $13(9.9 \%)$ \\
Septic shock & $11(8.4 \%)$ \\
Acute myocardial injury & $9(6.9 \%)$ \\
In-hospital mortality & $15(11.5 \%)$ \\
\hline
\end{tabular}

syndrome (SARS) and Middle East respiratory syndrome (MERS) (Tsang et al. 2003; Assiri et al. 2013).

Prompt and accurate diagnosis is the first and vital step to effectively control the ongoing outbreak of emerging COVID-19 epidemics (Sridhar et al. 2015; Yang et al. 2020). The radiological characteristics of patients with COVID-19 pneumonia are diverse, from nondistinctive features, diffuse changes, to destruction of the pulmonary parenchyma (Shi et al. 2020). However, the evidences based on radiological characteristics alone are not sufficient to confirm the virus. One commonly accepted clinical method in confirming infected cases of COVID-19 is based on detection of unique sequences of virus RNA via quantitative reverse transcriptase polymerase-chain-reaction (qRT-PCR), which has the advantages of high-accuracy and high specificity.

However, there is still a possibility that false-negative results might occur due to the low viral loads of the samples (To et al. 2020; Zou et al. 2020). Measurement of antiSARS-CoV-2 antibodies theoretically could remedy the detection of nucleic acid and be used for retrospective diagnosis and contact tracing. Therefore, in the present study, we retrospectively analyzed the sensitivities and dynamics of IgG and IgM antibodies detected by ELISA in COVID-19 patients at early stage of outbreak in Wuhan, as to provide early diagnosis information in this field.

\section{Materials and Methods}

\section{Study Design and Participants}

This single-center, retrospective study was conducted at Jin-Yin-Tan Hospital (Wuhan, China), which is a designated hospital to treat COVID-19 patients. We recruited 131 patients who had been diagnosed with COVID-19 before 15 January 2020. According to WHO interim guidance, at least one-time antibody detection was done during their hospitalization (WHO 2020b).

\section{Serological Tests for Anti-SARS-CoV-2 Antibodies}

Anti-SARS-CoV-2 IgG and IgM antibodies in serum samples of COVID-19 patients were detected with the serological methods as reported previously, which showed no crossreactivity with other commonly circulating human coronaviruses (e.g. HCoV-OC43) (Kissler et al. 2020). In-house anti-SARS-CoV-2 IgG and IgM ELISA kits were developed using SARSr-CoV Rp3 nucleocapsid protein (NP) as antigen, which is $>90 \%$ amino acid identity compared to reported SARSr-CoVs (Wang et al. 2018; Zhou et al. 2020a). For IgG test, MaxiSorp Nunc-immuno 96 well ELISA plates were coated with $100 \mathrm{ng} /$ well of recombinant NP overnight, then incubated with human sera in duplicates at a dilution of 1:20 for an hour at $37^{\circ} \mathrm{C}$, and followed by detection with anti-Human IgG-HRP conjugated monoclonal antibody (Kyab Biotech Co., Ltd, Wuhan, China) at a dilution of 1:40,000. For IgM test, MaxiSorp Nunc-immuno 96 well ELISA plates were coated with 500 ng/well anti-human IgM ( $\mu$ chain) overnight, then incubated with human sera in duplicates at $1: 100$ dilution for $40 \mathrm{~min}$ at $37^{\circ} \mathrm{C}$, and followed by detection with anti-Rp3 NP-HRP conjugated antibody (Kyab Biotech Co., Ltd, Wuhan, China) at a dilution of 1:4000. The OD values of 450-630 nm were calculated. 240 random negative control samples and two SARS-CoV-2 positive control samples were used to set the cutoff values of IgG and IgM. According to the Kit instruction, we used the mean OD value of the negative control samples plus three standard deviations to set the cutoff values of $\operatorname{IgG}$ and IgM at 0.143 and 0.142 , respectively. The specificity of these positive samples was confirmed by Western blot with recombinant Rp3 NP. 
Table 2 Laboratory parameters.

\begin{tabular}{ll}
\hline Parameter & \\
\hline White blood cell count, $\times 10^{9} / \mathrm{L}$ & $6.02 \pm 3.30$ \\
$<3.5$ & $25 / 131(19.1 \%)$ \\
$3.5 \sim 9.5$ & $88 / 131(67.2 \%)$ \\
$>9.5$ & $18 / 131(13.7 \%)$ \\
Neutrophil count, $\times 10^{9} / \mathrm{L}$ & $4.66 \pm 3.38$ \\
Lymphocyte count, $\times 10^{9} / \mathrm{L}$ & $1.00 \pm 0.52$ \\
$<1.1$ & $83 / 131(63.4 \%)$ \\
$\geq 1.1$ & $48 / 131(36.6 \%)$ \\
C-reactive protein, $\mathrm{mg} / \mathrm{L}$ & \\
$<5$ & $21 / 128(16.4 \%)$ \\
$\geq 5$ & $107 / 128(83.6 \%)$ \\
Procalcitonin, $\mathrm{ng} / \mathrm{mL}$ & \\
$<0.5$ & $123 / 128(96.1 \%)$ \\
$\geq 0.5$ & $5 / 128(3.9 \%)$ \\
ESR, mm/h & $49.82 \pm 5.06$ \\
$<15$ & $7 / 127(5.5 \%)$ \\
$\geq 15$ & $120 / 127(94.5 \%)$ \\
Interleukin6, pg/mL & $8.10 \pm 5.80$ \\
$<7$ & $48 / 97(49.5 \%)$ \\
$\geq 7$ & $49 / 97(50.5 \%)$ \\
Ferritin, ng/mL & \\
$<274.66$ & $33 / 119(27.7 \%)$ \\
$\geq 274.66$ & $86 / 119(72.3 \%)$ \\
LDH, mmol/L & $326.14 \pm 113.74$ \\
$<250$ & $43 / 128(33.6 \%)$ \\
$\geq 250$ & $85 / 128(66.4 \%)$ \\
FIB, g/L & $5.26 \pm 1.91$ \\
$<2$ & $3 / 124(2.4 \%)$ \\
$2 \sim 4$ & \\
$\geq 4$ & \\
D-Dimer, mg/L & \\
$<1.5$ & \\
\hline Co & \\
& \\
&
\end{tabular}

Continuous data are expressed as mean $\pm \mathrm{SD}$. Categorical data are presented as $\mathrm{n} / \mathrm{N}(\%)$, where $\mathrm{N}$ is the total number of patients with available data.

\section{Collection of Clinical Data and Evaluation of Chest CT}

All the clinical data on epidemiology, including exposure history, symptoms, underlying comorbidities and laboratory results were retrospectively extracted from electronic medical records. The date of disease onset was defined as the day when the symptom was noticed. All CT images were reviewed by two experienced radiologists, and decisions were reached by consensus agreement.

\section{Statistical Methods}

All statistical analyses were performed with SPSS software (version 20.0, IBM, Armonk, NY). Continuous normally distributed variables were presented as $\bar{x} \pm \mathrm{SD}$, non-normally distributed data and categorical variables are shown as frequencies and percentages. Statistical analyses were done by one-way analysis of variance (ANOVA) for numerical data and Chi Square Test for multiple comparisons of categorical data. $P$ value of less than 0.05 was considered statistically significant.

\section{Results}

\section{Clinical Characteristics}

Table 1 presents the demographic and clinical characteristics of the 131 confirmed cases (69\% male, $31 \%$ female), who were admitted to Jin-Yin-Tan Hospital between 30 December, 2019 and 15 January, 2020 with mean $9( \pm 3.9)$ days after onset of symptom. The average age was $51.4( \pm$ 11.8) years and $17.5 \%$ patients were older than 60. All patients were residents of Wuhan or surrounding areas. 75 (57\%) patients had a history of exposure to the Huanan Seafood Market, 12 (9\%) cases had exposure to patients with confirmed or highly suspected COVID-19, and 9 (7\%) patients were clustered onset. Among those 131 patients, $55(42 \%)$ had underlying chronic diseases, including 31 (24\%) hypertension, 14 (11\%) diabetes and 7 (5\%) with chronic heart disease.

The most common symptoms at admission were fever (124, 94.7\%), cough (96, 73.3\%) and dyspnea (50, 38.2\%). A few patients presented atypical onset symptoms, including headache $(15,12 \%)$, dizziness $(5,4 \%)$, nausea $(5,4 \%)$, vomit $(3,2 \%)$ and diarrhea $(2,1.5 \%)$. Almost half of the patients $(64,49 \%)$ received glucocorticoids, 19 (14.5\%) cases received human immunoglobulin; 33 $(25.5 \%)$ patients were treated with high-flow nasal cannula, $23(17.6 \%)$ with mechanical ventilation, 4 (3.1\%) with extracorporeal membrane oxygenation (ECMO), 6 (4.6\%) with renal replacement therapy, and $4(3.1 \%)$ with vasoconstrictive agents.

More than half $(72,55.5 \%)$ of the patients had damage in organ function, including $48(36.6 \%)$ with liver dysfunction, 40 (30.5\%) with ARDS, 13 (9.9\%) with acute kidney injury, 11 (8.4\%) with septic shock, and $9(6.9 \%)$ with acute cardiac injury. Most of the patients $(82,62.6 \%)$ were severe cases and a quarter of patients were critical cases according to the clinical classification defined by General Office of National Health Committee of China (General Office of National Health Committee 2020). 
Table 3 Differential sensitivity of ELISA for detection of IgM and IgG in different periods after disease onset.

\begin{tabular}{llllll}
\hline $\begin{array}{l}\text { Days after } \\
\text { onset }\end{array}$ & $\begin{array}{l}\text { Number of } \\
\text { samples }\end{array}$ & $\begin{array}{l}\text { Number of positive } \\
\text { for IgM by ELISA }\end{array}$ & $\begin{array}{l}\text { Number of positive } \\
\text { for IgG by ELISA }\end{array}$ & $\begin{array}{l}\text { ELISA OD ratio } \\
\text { of IgM }\end{array}$ & $\begin{array}{l}\text { ELISA OD ratio } \\
\text { of IgG }\end{array}$ \\
\hline $5 \sim 10$ & 34 & $13(38.2 \%)$ & $22(64.7 \%)^{*}$ & $0.202 \pm 0.273$ & $0.905 \pm 0.808$ \\
$11 \sim 20$ & 151 & $115(76.2 \%)$ & $147(97.4 \%)^{*}$ & $0.431 \pm 0.534$ & $1.683 \pm 0.653^{\#}$ \\
$21 \sim 30$ & 35 & $28(80.0 \%)$ & $35(100 \%)^{*}$ & $0.435 \pm 0.493$ & $1.686 \pm 0.542^{\#}$ \\
$31 \sim 40$ & 6 & $4(66.7 \%)$ & $5(83.3 \%)$ & $0.187 \pm 0.103$ & $1.621 \pm 0.932^{\#}$ \\
$5 \sim 40$ & 226 & $160(70.8 \%)$ & $209(92.5 \%)^{*}$ & $0.391 \pm 0.496$ & $1.565 \pm 0.722^{\#}$ \\
\hline
\end{tabular}

$* P<0.05$ versus $\operatorname{IgM}$ in the same period.

${ }^{\#} P<0.05$ versus 5 to 10 days.

Table 4 Differential sensitivity of ELISA for detection of IgM and IgG with different times in COVID-19 patients.

\begin{tabular}{llll}
\hline Times of detection & Number of patients & Number of positive for IgM by ELISA & Number of positive for IgG by ELISA \\
\hline 1 & 36 & $26(72.2 \%)$ & $35(97.2 \%)$ \\
2 & 95 & $87(91.6 \%)^{*}$ & $94(98.9 \%)$ \\
Total & 131 & $113(86.3 \%)$ & $129(98.5 \%)$ \\
\hline
\end{tabular}

$* P<0.05$ versus once.

Fifteen $(11.5 \%)$ of those patients died during hospitalization (Table 1).

\section{Laboratory Parameters}

The majority of the patients had a normal white blood cell count $(88 / 131,67.2 \%)$ and normal procalcitonin $(123 / 128$, $96.1 \%$ ). More than half of the patients had a reduced lymphocyte count $(83 / 131,63.4 \%)$, and increased indicators of inflammation, including ESR (120/127, 94.5\%), C-reactive protein $(107 / 128,83.6 \%)$, interleukin 6 (49/97, $50.5 \%)$, LDH $(85 / 128,66.4 \%)$ and ferritin $(86 / 119$, $72.3 \%$ ). Some patients showed abnormal coagulation function index, including increased fibrinogen (92/124, $74.2 \%)$ and increased D-Dimer (20/123, 16.3\%) (Table 2).

\section{CT Image Acquisition}

All patients had been chest CT scanned at outpatients department or at other hospitals before admission. All of them were reported with lung infection and, most likely, the viral pneumonia. 124 cases were re-conducted chest CT scan during hospitalization after 15 ( \pm 5.4$)$ days from onset. All chest CT images showed abnormalities, ground glass opacity and infiltrates shadows or consolidation. The majority of the patients presented bilateral, multifocal lung lesions, with peripheral distribution. Pleural effusion were uncommon imaging findings in those patients.

\section{Sensitivity of IgM and IgG ELISA Detection in COVID-19 Patients}

The sensitivities of the IgG and IgM ELISA detection in serum samples obtained from patients at different periods after disease onset are shown in Table 3. The overall sensitivities of $\operatorname{IgG}$ ELISA (92.5\%) were significantly higher than that of IgM ELISA $(70.8 \%)(P<0.001)$. In addition, the sensitivity of IgG ELISA in different periods after disease onset (5-10, 11-20 and 31-40 days) were generally higher than that of IgM ELISA, except for the equivalent from 31 to 40 days $(P>0.05)$ (Table 3$)$. The mean OD450 values of IgM and IgG for 226 serum samples obtained from the 131 confirmed COVID-19 cases were 0.391 and 1.565 , with standard deviations of 0.496 and 0.722 respectively (Table 3 ). For $\mathrm{IgG}$, the mean OD450 values significantly increased to 1.683 during 11 to 20 days after onset, and then maintained relatively high (Table 3). For IgM, however, the OD450 values reached the peak of 0.435 during 21 to 30 days after onset and fell back to 0.187 during 31 to 40 days after onset (Table 3). The sensitivity of IgM increased if the samples were redetected (Table 4).

\section{Dynamic Changes of IgG and IgM Antibodies for COVID-19 Patients}

Among the 131 confirmed cases, 16 cases were in hospital varying 30 to 60 days after onset of illness, whereas 109 
Table 5 Dynamic changes of IgM and IgG in COVID-19 patients $(\mathrm{n}=95)$.

\begin{tabular}{|c|c|c|c|c|}
\hline & Negative to positive & Positive to negative & Positive twice & Negative twice \\
\hline Number $(\%)$ of IgM change & $30(31.6 \%)$ & $10(10.5 \%)$ & $47(49.5 \%)$ & $8(8.4 \%)$ \\
\hline Number $(\%)$ of IgG change & $11(11.6 \%)$ & $3(3.2 \%)$ & $80(84.1 \%)$ & $1(1.1 \%)$ \\
\hline
\end{tabular}

patients were discharged within 30 days after onset. 115 patients survived. The median OD450 results from the available serum specimens of all patients were calculated and were plotted as a function of the days from onset of symptoms (Fig. 1). For IgM, the median OD450 rose above the baseline level at day 6 (seroconversion time), peaked at around day 18, and fell to below the baseline level at about day 36. The seroconversion time of $\mathrm{IgG}$ was 3 days. The IgG antibody level peaked at around 23 days, and then maintained relatively high (Fig. 1). IgG antibodies in 3 of 95 patients converted from positive to negative after 5 days (detected twice) (Table 5). Two of these three patients had only mild symptom but the third one presented critical situation and later died in hospital.

\section{Discussion}

Being able to detect the virus promptly and accurately is crucial in curbing the wide spread of infectious diseases, such as the COVID-19 which spread rapidly and killed a lot of people. Generally, the testing methods are based on the epidemiological risk, clinical features, imaging features and laboratory assays. We reported the retrospective sensitivity comparison of IgM and IgG ELISA detection for 131 confirmed cases of COVID-19 at early stage of outbreak in Wuhan. Meanwhile, the clinical characteristics of abovementioned patients were collected and analyzed. Over half of the patients in our study were male and had a history of exposure to the Huanan Seafood Market. The clinical features of fever, dry cough and dyspnea, which were in general the typical respiratory symptom and similar to SARS-CoV and MERS-CoV infections (Assiri et al. 2013), were respectively observed among 94.7\%, 73.3\% and $38.2 \%$ of the total 131 confirmed cases. However, a few patients presented common atypical onset symptoms, including headache, dizziness, nausea, vomit or diarrhea, which were unique and might lead to misdiagnosis. Moreover, since COVID-19 patients may harbor the virus in the intestine at the early or late stage of infection (Zhang et al. 2020), the suspected cases who mainly presented intestinal signs and symptoms were suggested to have a test with SARS-CoV-2 nucleic acid from faecal samples or anal swabs.

Among the total investigated cases, $55.5 \%$ of them suffered from organ function damage, and $63.4 \%$ of them
Fig. 1 Longitudinal profile of $\mathrm{IgG}$ and IgM antibodies in 131 patients with COVID-19.

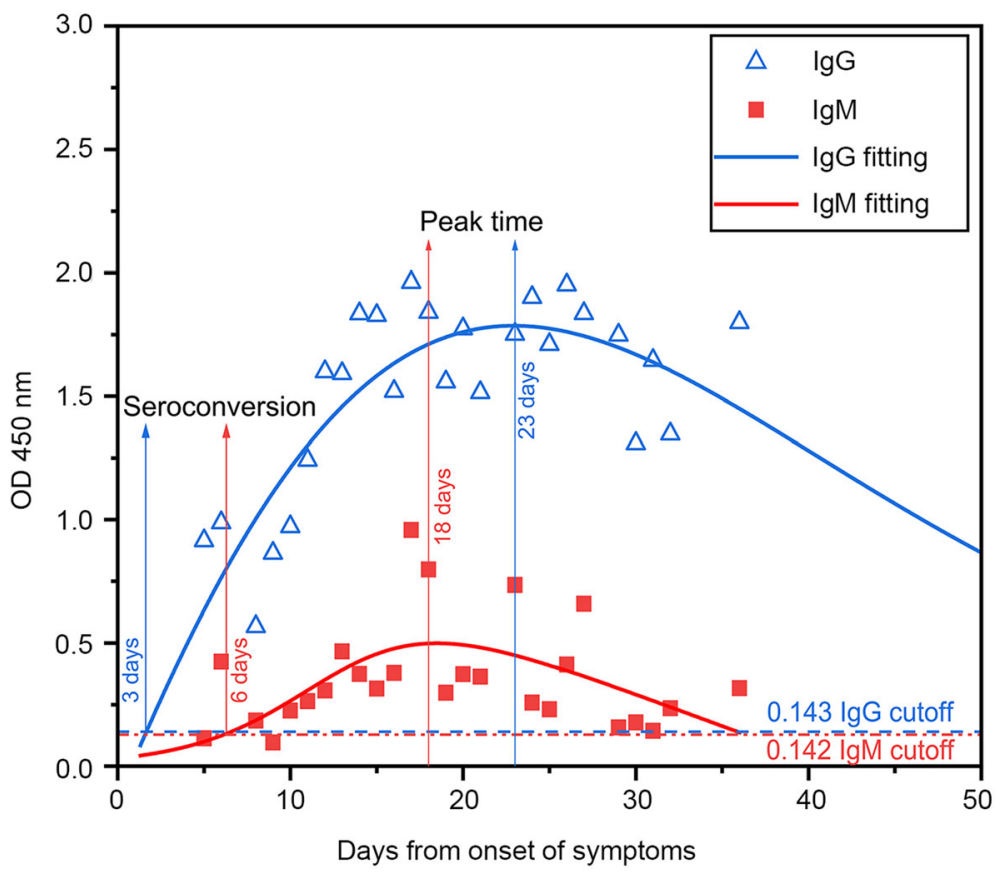


showed common symptoms on reduced lymphocyte count and increased indicators of inflammation. These are all indicators of critical illness and poor prognosis (Yang et al. 2020; Zhao et al. 2020). The majority of the patients had a normal white blood cell count $(67.2 \%$ of patients) and normal procalcitonin ( $92.1 \%$ of patients), which was consistent with rare bacterial infection. Unfortunately, 16 cases (12\%) developed severe illness and eventually died.

The sensitivity of IgG was significantly higher than that of IgM in this work, which is consistent with the results from recent reports (Long et al. 2020; Qu et al. 2020; Xiang et al. 2020; Zhao et al. 2020), where IgG tests performed $100 \%$ of sensitivity. The seroconversion of $\operatorname{IgG}$ against SARS-CoV-2 was earlier than that of IgM (3 days vs 6 days after onset), then maintained at high level longer than IgM. IgG maintained positive longer than 50 days, but IgM converted to negative around 36 days after onset. This observation is significantly different with dynamic changes of antibodies against SARS-CoV reported previously (Chan et al. 2007).

As we observed, IgG antibody could generally keep positive for a long period. While a surprising observation is that 3 cases converted from positive to negative after 5 days with twice tests. It is still unclear whether the COVID-19 patients would acquire permanent immunity to this disease after certain time. Further studies are needed to confirm this.

In conclusion, our findings suggested that detection of antibodies showed tremendous value in helping diagnosis of COVID-19 infection. IgG could be a sensitive indicator for retrospective diagnosis and contact tracing, and IgM could be an indicator of early COVID-19.

Acknowledgements We acknowledge Dr. Rui Shu from Linköping University, Sweden for deep discussion.

Author Contributions HS and SW designed the study and wrote the paper. SR, YW, JZ, YW and RL collected the data. YY, HL, SP, and YO analyzed the data. SY, PZ and YS checked and finalized the manuscript. All authors read and approved the final manuscript to be published.

\section{Compliance with Ethical Standards}

Conflict of interest The authors declare that they have no conflict of interest.

Animal and Human Rights Statement This study was approved by the Ethics Commission of Jin-Yin-Tan Hospital (KY-2020-47.01). Written informed consent was waived by the Ethics Commission of the designated hospital according to the policy for public health outbreak investigation of emerging infectious diseases.

\section{References}

Assiri A, Al-Tawfiq JA, Al-Rabeeah AA, Al-Rabiah FA, Al-Hajjar S, Al-Barrak A, Flemban H, Al-Nassir WN, Balkhy HH, AlHakeem RF, Makhdoom HQ, Zumla AI, Memish ZA (2013) Epidemiological, demographic, and clinical characteristics of 47 cases of Middle East respiratory syndrome coronavirus disease from Saudi Arabia: a descriptive study. Lancet Infect Dis 13:752-761

Chan KH, Sonnenberg K, Niedrig M, Lam SY, Pang CM, Chan KM, Ma SK, Seto WH, Peiris JSM (2007) Use of antibody avidity assays for diagnosis of severe acute respiratory syndrome coronavirus infection. Clin Vaccine Immunol 14:1433-1436

Chen N, Zhou M, Dong X, Qu J, Gong F, Han Y, Qiu Y, Wang J, Liu Y, Wei Y, Xia J, Yu T, Zhang X, Zhang L (2020) Epidemiological and clinical characteristics of 99 cases of 2019 novel coronavirus pneumonia in Wuhan, China: a descriptive study. Lancet 395:507-513

General Office of National Health Committee (2020) Office of state administration of traditional Chinese medicine. Notice on the issuance of a program for the diagnosis and treatment of novel coronavirus (2019-nCoV) infected pneumonia (trial version 6)

Huang C, Wang Y, Li X, Ren L, Zhao J, Hu Y, Zhang L, Fan G, Xu J, Gu X, Cheng Z, Yu T, Xia J, Wei Y, Wu W, Xie X, Yin W, Li H, Liu M, Xiao Y, Gao H, Guo L, Xie J, Wang G, Jiang R, Gao Z, Jin Q, Wang J, Cao B (2020) Clinical features of patients infected with 2019 novel coronavirus in Wuhan, China. Lancet 395:497-506

Kissler SM, Christine T, Edward G, Yonatan HG, Marc L (2020) Projecting the transmission dynamics of SARS-CoV-2 through the postpandemic period. Science 368:860-868

Long QX, Liu BZ, Deng HJ, Wu GC, Deng K, Chen YK, Liao P, Qiu JF, Lin Y, Cai XF, Wang DQ, Hu Y, Ren JH, Tang N, Xu YY, Yu LH, Mo Z, Gong F, Zhang XL, Tian WG, Hu L, Zhang XX, Xiang JL, Du HX, Liu HW, Lang CH, Luo XH, Wu SB, Cui XP, Zhou Z, Zhu MM, Wang J, Xue CJ, Li XF, Wang L, Li, ZJ, Wang K, Niu CC, Yang QJ, Tang XJ, Zhang Y, Liu XM, Li JJ, Zhang DC, Zhang F, Liu P, Yuan J, Li Q, Hu JL, Chen J, Huang, AL (2020) Antibody responses to SARS-CoV-2 in patients with COVID-19. Nat Med 1-4

Qu J, Wu C, Li XY, Zhang G, Jiang Z, Li X, Zhu Q, Liu L (2020) Profile of IgG and IgM antibodies against severe acute respiratory syndrome coronavirus 2 (SARS-CoV-2). Clin Infect Dis, Accepted

Shi H, Han X, Jiang N, Cao Y, Alwalid O, Gu J, Fan Y, Zheng C (2020) Radiological findings from 81 patients with COVID-19 pneumonia in Wuhan, China: a descriptive study. Lancet Infect Dis 20:425-434

Sridhar S, To KKW, Chan JFW, Lau SKP, Woo PCY, Yuen KY (2015) A systematic approach to novel virus discovery in emerging infectious disease outbreaks. J Mol Diagn 17:230-241

To KKW, Tsang OTY, Leung WS, Tam AR, Wu TC, Lung DC, Yip CCY, Cai JP, Chan JMC, Chik TSH, Lau DPL, Choi CYC, Chen LL, Chan WM, Chan KH, Ip JD, Ng ACK, Poon RWS, Luo CT, Cheng VCC, Chan JFW, Hung IFN, Chen Z, Chen H, Yuen KY (2020) Temporal profiles of viral load in posterior oropharyngeal saliva samples and serum antibody responses during infection by SARS-CoV-2: an observational cohort study. Lancet Infect Dis 20:565-574

Tsang KW, Ho PL, Ooi GC, Yee WK, Wang T, Chan-Yeung M, Lam WK, Seto WH, Yam LY, Cheung TM, Wong PC, Lam B, Ip MS, Chan J, Yuen KY, Lai KN (2003) A cluster of cases of severe acute respiratory syndrome in Hong Kong. N Engl J Med 348:1977-1985 
Wang D, Hu B, Hu C, Zhu F, Liu X, Zhang J, Wang B, Xiang H, Cheng Z, Xiong Y, Zhao Y, Li Y, Wang X, Peng Z (2020) Clinical characteristics of 138 hospitalized patients with 2019 novel coronavirus-infected pneumonia in Wuhan, China. JAMA 323:1061-1069

Wang N, Li S-Y, Yang X-L, Huang H-M, Zhang Y-J, Guo H, Luo C-M, Miller M, Zhu G, Chmura AA, Hagan E, Zhou J-H, Zhang Y-Z, Wang L-F, Daszak P, Shi Z-L (2018) Serological Evidence of Bat SARS-Related Coronavirus Infection in Humans, China. Virol Sin 3:104-107

World Health Organization (2020a) Coronavirus disease (COVID-19) situation report, 170. Accessed 8 July 2020

World Health Organization (2020b) Clinical management of severe acute respiratory infection when novel coronavirus (2019-nCoV) infection is suspected: interim guidance, 28 January 2020. World Health Organization

Xiang F, Wang X, He X, Peng Z, Yang B, Zhang J, Zhou Q, Ye H, Ma Y, Li H, Wei X, Cai P, Ma WL (2020) Antibody detection and dynamic characteristics in patients with COVID-19. Clin Infect Dis, Accepted

Yang X, Yu Y, Xu J, Shu H, Xia J, Liu H, Wu Y, Zhang L, Yu Z, Fang M, Yu T, Wang Y, Pan S, Zou X, Yuan S, Shang Y (2020) Clinical course and outcomes of critically ill patients with SARS-CoV-2 pneumonia in Wuhan, China: a single-centered, retrospective, observational study. Lancet Respir Med 8:475-481

Zhang W, Du RH, Li B, Zheng XS, Yang XL, Hu B, Wang Y, Xiao GF, Yan B, Shi ZL, Zhou P (2020) Molecular and serological investigation of 2019-nCoV infected patients: implication of multiple shedding routes. Emerg Microbes Infect 9:386-389

Zhao J, Yuan Q, Wang H, Liu W, Liao X, Su Y, Wang X, Yuan J, Li T, Li J, Qian S, Hong C, Wang F, Liu Y, Wang Z, He Q, Li Z,
He B, Zhang T, Fu Y, Ge S, Liu L, Zhang J, Xia N, Zhang Z (2020) Antibody responses to SARS-CoV-2 in patients of novel coronavirus disease 2019. Clin Infect Dis, Accepted

Zhou F, Yu T, Du R, Fan G, Liu Y, Liu Z, Xiang J, Wang Y, Song B, Gu X, Guan L, Wei Y, Li H, Wu X, Xu J, Tu S, Zhang Y, Chen H, Cao B (2020) Clinical course and risk factors for mortality of adult inpatients with COVID-19 in Wuhan, China: a retrospective cohort study. Lancet 395:1054-1062

Zhou P, Yang XL, Wang XG, Hu B, Zhang L, Zhang W, Si HR, Zhu Y, Li B, Huang CL, Chen HD, Chen J, Luo Y, Guo H, Jiang RD, Liu MQ, Chen Y, Shen XR, Wang X, Zheng XS, Zhao K, Chen QJ, Deng F, Liu LL, Yan B, Zhan FX, Wang YY, Xiao G, Shi ZL (2020a) Discovery of a novel coronavirus associated with the recent pneumonia outbreak in humans and its potential bat origin. bioRxiv 2020.01.22.914952

Zhou P, Yang XL, Wang XG, Hu B, Zhang L, Zhang W, Si HR, Zhu Y, Li B, Huang CL, Chen HD, Chen J, Luo Y, Guo H, Jiang RD, Liu MQ, Chen Y, Shen XR, Wang X, Zheng XS, Zhao K, Chen QJ, Deng F, Liu LL, Yan B, Zhan FX, Wang YY, Xiao GF, Shi ZL (2020b) A pneumonia outbreak associated with a new coronavirus of probable bat origin. Nature 579:270-273

Zhu N, Zhang D, Wang W, Li X, Yang B, Song J, Zhao X, Huang B, Shi W, Lu R, Niu P, Zhan F, Ma X, Wang D, Xu W, Wu G, Gao GF, Tan W (2020) A novel coronavirus from patients with pneumonia in China, 2019. N Engl J Med 382:727-733

Zou L, Ruan F, Huang M, Liang L, Huang H, Hong Z, Yu J, Kang M, Song Y, Xia J, Guo Q, Song T, He J, Yen HL, Peiris M, Wu J (2020) SARS-CoV-2 viral load in upper respiratory specimens of infected patients. N Engl J Med 382:1177-1179 